I NVESTI GACI ÓN

\title{
El precio del cigarrillo y la reducción del consumo en Cuba
}

\author{
The price of cigarettes and the reduction of smoking in Cuba
}

\author{
DraC. Nery Suárez Lugo \\ Escuela Nacional de Salud Pública. La Habana, Cuba.
}

\section{RESUMEN}

Introducción: el estudio del consumo de cigarrillos que se ejecuta con periodicidad anual, constituye una investigación oportuna, poco costosa y confiable para seguir y perfeccionar el Programa de Prevención y Control del Tabaquismo.

Objetivos: describir el consumo en el año 2010, su relación con precio y formas de comercialización, la elasticidad de la demanda y otros aspectos económicos y sociales.

Métodos: se realizó un estudio observacional descriptivo, con datos agregados de series temporales.

Resultados: en relación con el 2009 el consumo per cápita de cigarrillos decreció $12,0 \%$, el precio promedio aumentó $7,97 \%$ y las ventas solo aumentaron en el mercado liberado en pesos convertibles. La demanda de cigarrillos presentó elasticidad, por lo que la cantidad demandada disminuirá proporcionalmente más de lo que aumenta el precio.

Conclusiones: en el año 2010 la relación entre precio y consumo se encuentra presente también en el contexto cubano y la elasticidad de la demanda de cigarrillos se verá más beneficiada en el año 2011 al no existir la oferta de cigarrillos normados, situación que debe variar en los años siguientes si no se producen nuevos incrementos del precio y se refuerza el componente educativo en la estrategia de prevención y control del tabaquismo.

Palabras clave: Tabaquismo, precio, consumo, Cuba. 
ABSTRACT

Introduction: the annual study of the consumption of cigarettes is a timely, lowcost and reliable research to follow and upgrade the Program of Prevention and Control of Smoking.

Objectives: to describe the consumption of cigarettes in 2010, its association with the price and the forms of commercialization, the elasticity of demand and other economic and social aspects.

Methods: a descriptive and observational study was performed, using time series added data.

Results: when compared with the 2009 consumption, the per capita consumption of cigarettes in 2010 decreased by $12 \%$, the average price rose by $7.97 \%$ and the sales increased only in the market operating with convertible pesos. The cigarette demand was elastic, so the reduction in the demanded quantity will be proportionally higher than the price increase.

Conclusions: the price-consumption ratio in 2010 was also present in the Cuban setting, and the elasticity of cigarette demand will be more benefited in 2011 since the cigarette supply based on the rationing card ceased to exist. This situation may change in the following years if new price increases are not implemented and the educational work included in the smoking prevention and control strategy is not reinforced.

Key words: Smoking, price, consumption, Cuba.

\section{NTRODUCCI ÓN}

La economía y la salud pública son dos disciplinas que tienen influencia sobre las políticas públicas pero con visiones distintas acerca de una misma cuestión, que generalmente trascienden a temas relacionados con la ética.

Desde una perspectiva económica, fumar se considera como parte de un "estilo de vida" como puede ser preferir el consumo de un tipo de alimento o la práctica de deportes de riesgo. Para que el fumar sea un problema social debe dañar a otros individuos o entrañar peligros desconocidos para el que lo pone en práctica. Como el consumidor conoce y paga conscientemente el costo de su elección de fumar, no puede ser catalogado como "fallo de mercado" y por consiguiente, no hay necesidad de intervención pública.

Desde la perspectiva de la salud pública, la posición es bien diferente. El tabaquismo es factor de riesgo de las principales causas de mortalidad y morbilidad, produce daños a la salud del individuo fumador y también al fumador pasivo, pero puede existir o no conciencia de la nocividad del tabaquismo derivado del supuesto de una disponibilidad de información desigual, dado el peso generalmente superior de la publicidad, muchas veces engañosa, en relación con las campañas educativas.

Es por consiguiente responsabilidad de la salud pública la intervención para la prevención y control del tabaquismo, aunque en ocasiones no cuente con todas las características de un "fallo de mercado" desde la perspectiva económica. ${ }^{1}$

http://scielo.sld.cu 
Un reflejo de lo anterior es que en Cuba el debate sobre la relación entre los costos y los beneficios del tabaquismo es un punto, que aunque aparece con frecuencia en las agendas de discusión de los que se encargan de la prevención y promoción de la salud, no se ha podido colocar de manera adecuada en la agenda de los que deciden las políticas públicas que de una forma u otra repercuten en la salud.

Sin embargo, por las particularidades del contexto cubano, donde los recursos del Estado se encuentran centralizados, este es un tema que posee además de una importante connotación ética, la económica. Debido a los costos que el tabaquismo implica a la salud pública y al medio ambiente, los beneficios que proporciona a la economía nacional mediante la creación de empleos, recaudación de impuestos e ingresos en moneda libremente convertible, pueden quedar anulados. ${ }^{2}$

Esta connotación ética se ve más aún cuando se analiza que en una sociedad que promueve la salud se valore la importancia de la recaudación de ingresos a partir de productos que afectan la salud individual y también la economía familiar, derivado no solo de los gastos por concepto de compra de cigarrillos, sino por los relacionados con la atención a la salud, pérdidas laborales e inclusive la reducción en la compra de alimentos de los fumadores y sus familias (Suárez Lugo N, Gálvez González AM, Caraballoso HM. Aspectos fundamentales para el perfeccionamiento de la estrategia para la prevención y control del tabaquismo en Cuba. Informe Técnico. La Habana. Escuela Nacional de Salud Pública. 2010).

Lo anterior puede avalarse por el hecho de que aquellos fumadores que consumen una cajetilla diaria, según los precios vigentes de la caja de cigarrillos en el año 2010, gastaron al mes 231,60 pesos, y los que fuman dos, 463,20 pesos. Esta última cifra es superior a 429 pesos, reconocida como ingreso medio. ${ }^{3}$ Lo anterior puede crearle a las familias fumadoras de menos ingresos, serias limitaciones en su calidad de vida.

Para la salud pública, el efecto del tabaquismo sobre la salud y el bienestar de los cubanos no pueden supeditarse al impacto económico y social. Tampoco establecer a nivel internacional una estrategia de desplazar el mercado del tabaco hacia aquellos países en que la ausencia de regulaciones de prohibición de fumar convierta a su población en vulnerable. ${ }^{4}$

El presente estudio ofrece argumentos para comprender la necesidad de que las medidas económicas resulten coherentes con las políticas de salud y ofrece elementos sobre la economía del tabaco, tanto por su papel dentro de la matriz económica del país como por las políticas relacionadas con su consumo, en un momento en que por primera vez, se toma la decisión de retirar los cigarrillos de la oferta normada, lo que tendrá impacto en el consumo de la población.

La implementación de esta decisión, ${ }^{5}$ ha estado más en relación con los ajustes económicos que se realizan en el país que como objetivo de salud, pues en este sentido las estrategias para prevenir y controlar adicciones, a pesar de su importancia enorme, son complejas y rinden frutos a largo plazo y en este caso, han estado compitiendo con otros problemas urgentes de la sociedad a los que los directivos y las instituciones de salud han tenido que dedicarle mayor tiempo y recursos, en las condiciones de una sociedad donde el tabaco se encuentra en un importante renglón de la economía. ${ }^{6}$

Los estudios de consumo y precio de cigarrillos, no son los más frecuentes en la literatura, sin embargo, constituyen un indicador de impacto a corto plazo, se ejecutan con pocos recursos y permite evaluar, de forma sistemática, los resultados que se obtienen con el Programa de Prevención y Control del Tabaquismo. ${ }^{7}$ 
Los resultados obtenidos en Cuba desde el año 1985, permiten disponer de una serie cronológica que ofrece una amplia gama de posibilidades de análisis y a la vez establecer relaciones con la prevalencia, incidencia del tabaquismo y la mortalidad y morbilidad asociadas al consumo de tabaco.

El consumo de cigarrillos y tabacos, su relación con la situación socioeconómica existente, las formas de comercialización y el precio, permiten a las autoridades sanitarias disponer de resultados cientificamente argumentados para tomar decisiones que respalden su reducción.

Es importante, por tanto, indagar sobre el consumo de cigarrillos en Cuba en el 2010 y su relación con el precio y formas de comercialización, así como determinar la elasticidad de la demanda del cigarrillo con respecto al precio, objetivos de este trabajo.

\section{MÉTODOS}

Se realizó un estudio observacional descriptivo, que utilizó la alternativa de fuente de datos agregados de series temporales, que permite análisis anuales con muy bajo costo.

Las variables estudiadas, los criterios seguidos para definir cada una y su forma de medición, aparecen a continuación:

- Consumo per cápita de cigarrillos: el indicador empleado para su medición es la cantidad de cigarrillos vendidos en el año dividido entre la población 15 años y más.

- Proporción de mercado: porcentaje de las ventas de cigarrillos que se realizan en cada tipo de mercado, su medición se efectúa al dividir el total de las ventas de cigarrillos vendidos en cada tipo de mercado entre el total de las ventas multiplicado por 100.

- Precio promedio: promedio del precio de la cajetilla de 20 cigarrillos de todas las marcas vendidas, medido por el valor total de las ventas dividido entre la cantidad de cajetillas de cigarrillos vendidos.

- Elasticidad de demanda: modificaciones que se producen en el consumo de cigarrillos derivado de cambios en el precio, su indicador se determina mediante la división del porcentaje de cambio en el consumo de cigarrillos entre el porcentaje de cambio en el precio de cigarrillos por 100.

Para la captura de datos se emplearon técnicas de análisis de información secundaria, entrevista individual, observación no participante y criterio de expertos.

Se determinaron los cigarrillos consumidos por adulto mayor de 15 años, calculado de las cifras de venta en moneda nacional y moneda libremente convertible. ${ }^{8,9}$

Toda la información de venta de los cigarrillos tuvo como fecha de cierre el 31 de diciembre del 2010.

http://scielo.sld.cu 
Se tomó la población de 15 años y más (9 278 387) estimada el 31 de diciembre de 2009 por la Oficina Nacional de Estadísticas. ${ }^{10}$ Aunque se disponía del estimado del 31 de diciembre del 2010, este no se encontraba desagregado por grupos de edades.

Referente a la elasticidad de la demanda con respecto al precio, se realizó la medición de la capacidad de respuesta de la cantidad demandada de cigarrillos a los cambios en el precio en los años 2009 y 2010, lo que equivale al porcentaje de cambio en la cantidad demandada dividido por el porcentaje de cambio en el precio. Si la elasticidad es menor a -1,0 la cantidad de cigarrillos demandada disminuirá proporcionalmente más de lo que aumenta el precio, mientras que se considerará que la demanda es inelástica si la elasticidad es mayor a $-1,0$ y la cantidad demandada disminuirá menos que el porcentaje de aumento en el precio. ${ }^{11}$

La metodología empleada para la captura de datos, procesamiento y análisis de la información se mantuvo de igual forma desde que se inició la realización de este tipo de estudio en la década del 80 del pasado siglo, por los especialistas del Instituto Cubano de Investigaciones y Orientación de la Demanda Interna (ICIODI), por lo que existe coherencia en la construcción de la serie cronológica que se presenta. ${ }^{12}$

\section{RESULTADOS}

\section{Consumo per cápita de cigarrillos}

En el año 2010 el consumo per cápita en la población cubana de 15 años y más fue de 1362,5 cigarrillos (tabla 1). Esta cifra equivale a que cada cubano en esas edades fumó aproximadamente 4 cigarrillos diarios. En el año 2010 en relación con el 2009, el consumo per cápita de cigarrillos decreció $12,0 \% ; 1,42 \%$ si se compara con el 2000 y 36,93 \% con el 1985. En el año 1985 se inició una tendencia decreciente del consumo de cigarrillos hasta el 2001 que se reviertió a creciente, con solo una discreta disminución en el 2005 para continuar en aumento en 2006 y hasta el 2008.

En el 2008 se registró el consumo per cápita de cigarrillos más alto desde el 1992, para iniciar de nuevo su descenso en el 2009 y presentar en el 2010 la cifra más baja en el decenio, aunque ligeramente superior a la del 2001 y una de las más bajas desde la década del 80 del pasado siglo. El decrecimiento del 2010 se produjo tomando como base la cifra de 5,67 \% que ya tuvo un descenso en el 2009.

\section{Mercado de cigarrillos}

El mercado de cigarrillos en el 2010 presentó un cambio muy importante, ya que como parte de las medidas que de forma gradual se vienen aplicando para limitar los subsidios en los que incurre el Estado, el Consejo de Ministros determinó suprimir la venta de los cigarrillos de la canasta familiar normada. Esta medida entró en vigor el 1ro. de septiembre de 2010, por lo que no estuvo presente en el mercado este tipo de oferta en el cuarto trimestre del año, aunque la distribución a organismos, denominada consumo social (entrega a organismos e instituciones para distribución gratuita o a precios bajos), se siguió realizando.

http://scielo.sld.cu 
Tabla 1. Consumo per cápita de cigarrillos*. Cuba, 1985, 1990-2010

\begin{tabular}{|c|c|}
\hline Año & Cigarrillos (unidades) \\
\hline 1985 & 2164 \\
\hline 1990 & 1934 \\
\hline 1991 & 1678 \\
\hline 1992 & 1664 \\
\hline 1993 & 1572 \\
\hline 1994 & 1660 \\
\hline 1995 & 1395 \\
\hline 1996 & 1295 \\
\hline 1997 & 1196 \\
\hline 1998 & 1382 \\
\hline 1999 & 1449 \\
\hline 2000 & 1382 \\
\hline 2001 & 1314 \\
\hline 2002 & 1425 \\
\hline 2003 & 1446 \\
\hline 2004 & 1501 \\
\hline 2005 & 1459 \\
\hline 2006 & 1470 \\
\hline 2007 & 1503 \\
\hline 2008 & 1641 \\
\hline 2009 & 1548 \\
\hline 2010 & 1363 \\
\hline
\end{tabular}

* Población 15 años y más.

En moneda nacional se ofertaron los cigarrillos de producción nacional destinados al consumo interno y en pesos cubanos convertibles los consignados a la exportación en fronteras así como otros de importación, que por su precio más elevado, de acuerdo a entrevistas realizadas a los vendedores, se mantuvo con muy poca demanda, al igual que en años anteriores.

De las ventas de cigarrillos, se realizó de manera liberada el 83,14 \%, de ellos $69,85 \%$ en moneda nacional y $13,29 \%$ en pesos convertibles, lo que aumentó la proporción general y de ambas formas de ventas liberadas, derivado del cese de la venta racionada (tabla 2 ).

De las ventas totales de cigarrillos se realizaron en el mercado normado $16,86 \%$, esto es, se adquirieron por la libreta de racionamiento o por el denominado consumo social. De ellos, el 85,31 \% correspondió al mercado normado y 14,69\% a la entrega a organismos. Estas proporciones presentaron variaciones muy discretas en relación con los años anteriores (tabla 3). 
Tabla 2. Ventas de cigarrillos en el mercado (\%) según forma de comercialización. Cuba $2000 / 2010$

\begin{tabular}{|l|c|c|c|c|c|}
\hline \multicolumn{1}{|l|}{} & & & & \\
\hline Forma de comercialización / año & 2000 & 2005 & 2008 & 2009 & 2010 \\
\hline Liberada CUP & & & & & \\
\hline Liberada CUC & 10,16 & 9,79 & 15,64 & 11,27 & 13,29 \\
\hline Liberada total & 62,04 & 70,73 & 76,90 & 76,30 & 83,14 \\
\hline Normada & 37,96 & 29,27 & 23,10 & 23,70 & 16,86 \\
\hline Total & 100 & 100 & 100 & 100 & 100 \\
\hline
\end{tabular}

Tabla 3. Ventas de cigarrillos en el mercado normado (\%). Cuba 2004/2010

\begin{tabular}{|l|c|c|c|c|c|c|}
\hline Distribución normada/año & 2004 & 2005 & 2008 & 2009 & 2010 \\
\hline Población & 87,54 & 86,01 & 88,26 & 88,83 & 85,31 \\
\hline Organismos & 12,46 & 13,09 & 11,74 & 11,17 & 14,69 \\
\hline Total & 100 & 100 & 100 & 100 & 100 \\
\hline
\end{tabular}

En la entrega a organismos, el Ministerio de Salud Pública representa el 17,79\% del total, superior a años anteriores y continúa ocupando el segundo lugar proporcional de esta forma de distribución.

Resulta interesante que la venta de los cigarrillos liberados en moneda nacional disminuyó $1,22 \%$, por lo que cambió la tendencia sostenida de un aumento que se mantuvo alrededor del $4 \%$ en el último quinquenio, aunque en el 2009 fue solo $0,13 \%$. Sin embargo, el análisis del valor de las ventas del año representa un incremento, lo que significa que los consumidores compraron con más dinero menos cantidad de cigarrillos debido a que los que se ofertaban a precios bajos en el mercado normado comenzaron a venderse a precios altos en el liberado en moneda nacional. ${ }^{8}$

La venta de los cigarrillos liberados en moneda libremente convertible aumentó $8,38 \%$, siguiendo su tendencia al aumento, pero sin sobrepasar los años anteriores. En valores también se produjo un incremento. Es de destacar que este crecimiento se realizó sobre un incremento acumulado que se puede considerar sostenido desde que se inició este tipo de oferta.

La venta de cigarrillos normados descendió $34,55 \%$, esta reducción equivale en los dos primeros cuatrimestre a la reducción natural derivada de la forma de distribución y en el cuarto trimestre a la supresión de este tipo de distribución a la población, quedando solo la de organismos (tabla 4). 
Tabla 4. Cambios en las ventas de cigarrillos en el mercado según forma de comercialización. Cuba 2009/2010

\begin{tabular}{|l|c|c|c|}
\hline \multicolumn{1}{|c|}{$\begin{array}{c}\text { Forma } \\
\text { de comercialización }\end{array}$} & Año 2010 & Año 2009 & $\%$ \\
\hline Normada & 2131,9 & 3256,9 & 34,55 \\
\hline Liberada CUP & 8830,7 & 8940,1 & 1,22 \\
\hline Liberada CUC & 1679,6 & 1549,6 & 8,38 \\
\hline \hline Total & 12642,2 & 13746,4 & 8,04 \\
\hline
\end{tabular}

En pesos cubanos se vendió el 19,45 \% de forma normada. De forma liberada, el $84,01 \%$ se entregó en moneda nacional y el 15,99 \% en pesos cubanos convertibles, con variación en relación con el 2009 en cuanto a la proporción en los dos mercados y el decrecimiento que se produjo de forma natural en el normado al cesar este en el último cuatrimestre del año (tabla 5).

Tabla 5. Ventas de cigarrillos en el mercado según forma de comercialización (\%). Cuba 2004/2010

\begin{tabular}{|l|c|c|c|c|c|c|}
\hline Forma de comercialización/año & 2004 & 2005 & 2008 & 2009 & 2010 \\
\hline Normada (población) & 32,80 & 32,81 & 24,97 & 26,70 & 19,45 \\
\hline Liberada CUP & 67,20 & 67,19 & 24,97 & 26,70 & 80,55 \\
\hline Total CUP & 100 & 100, & 100, & 100 & 100 \\
\hline Liberada CUP & 85,51 & 86,17 & 79,66 & 85,23 & 84,01 \\
\hline Liberada CUC & 14,49 & 13,83 & 20,34 & 14,77 & 15,99 \\
\hline Total & 100 & 100 & 100, & 100, & 100 \\
\hline
\end{tabular}

Aunque desde años anteriores se amplió y estabilizó la oferta de los cigarrillos en moneda nacional y estos tienen un menor precio en comparación con la venta en pesos cubanos convertibles, aún existe el criterio en los vendedores, de que los consumidores consideran que no es buena su calidad, lo que constituye una posible explicación a que aumente la venta en CUC y disminuya en CUP, aunque esta última aumentara en valor. ${ }^{8}$

En el 2010, de cada 100 cigarrillos que se vendieron, 17 correspondieron al mercado de precios bajos y 83 al de precios altos, de ellos, 70 se compraron en moneda nacional y 13 en libremente convertible.

También se mantuvo la oferta en moneda nacional de cigarrillos sueltos en la red de comercio minorista, con su correspondiente efecto de estimular el consumo en los jóvenes al abaratar el precio relativo del producto. Continúa la oferta de cajetillas con 10 cigarrillos para la venta en pesos convertibles que como estrategia de mercadeo cumple el mismo propósito. 


\section{Precio promedio del cigarrillo}

El precio promedio de la cajetilla de cigarrillos en el 2010 fue de 7,72 pesos CUP (tabla 6), lo que representó un incremento de 0,57 pesos CUP por cajetilla en relación con el año anterior. La equivalencia del precio promedio era de 0,32 pesos cubanos convertibles (CUC) y 0,38 dólares estadounidenses (USD).

Tabla 6. Estimado precio promedio cajetillas-cigarrillos CUP. Cuba 1960-2010

\begin{tabular}{|l|l|}
\hline Año & $\begin{array}{l}\text { Estimado } \\
\text { precio } \\
\text { promedio } \\
\text { cajetilla 20 } \\
\text { cigarrillos } \\
\text { CUP }\end{array}$ \\
\hline $1960 / 9$ & 0,20 \\
\hline 1970 & 0,62 \\
\hline 1973 & 0,88 \\
\hline 1978 & 0,93 \\
\hline 1980 & 0,99 \\
\hline 1985 & 1,15 \\
\hline 1990 & 1,19 \\
\hline 1991 & 1,19 \\
\hline 1992 & 2,40 \\
\hline 1993 & 2,42 \\
\hline 1994 & 4,60 \\
\hline 1995 & 4,65 \\
\hline 1996 & 4,80 \\
\hline 1997 & 4,85 \\
\hline 1998 & 5,20 \\
\hline 1999 & 5,35 \\
\hline 2000 & 5,60 \\
\hline 2001 & 6,10 \\
\hline 2002 & 6,68 \\
\hline 2003 & 6,84 \\
\hline 2004 & 7,15 \\
\hline 2005 & 6,55 \\
\hline 2006 & 6,81 \\
\hline 2007 & 6,93 \\
\hline 2008 & 7,35 \\
\hline 2009 & 7,15 \\
\hline 2010 & 7,72 \\
\hline
\end{tabular}


El porcentaje de cambio en el precio promedio del cigarrillo, al comparar el año 2010 con el año 2009, tuvo un incremento de 7,97 \% superior al que ha tenido desde el año 2002 (tabla 7).

Tabla 7. Cambio en el consumo per cápita y estimado precio promedio cajetilla-cigarrillos. Cuba 1985-2010

\begin{tabular}{|l|c|c|}
\hline Año & Consumo \% & Precio \% \\
\hline $2010 / 09$ & 11,4 & 7,97 \\
\hline $2009 / 08$ & 5,67 & 2,73 \\
\hline $2008 / 07$ & 9,18 & 6,06 \\
\hline $2007 / 06$ & 2,24 & 1,76 \\
\hline $2006 / 05$ & 0,75 & 3,96 \\
\hline $2005 / 04$ & 2,76 & 8,4 \\
\hline $2004 / 03$ & 3,8 & 4,53 \\
\hline $2003 / 02$ & 1,47 & 2,40 \\
\hline $2002 / 01$ & 8,44 & 9,5 \\
\hline $2001 / 00$ & 4,93 & 8,9 \\
\hline $2000 / 99$ & 4,63 & 4,67 \\
\hline $1999 / 90$ & 25,08 & 22,24 \\
\hline $1990 / 85$ & 10,7 & 1,03 \\
\hline $2005 / 2001$ & 11,03 & 7,37 \\
\hline $2005 / 85$ & $(32,58)$ & 569,56 \\
\hline $2010 / 00$ & $(0,80)$ & 37,85 \\
\hline $2010 / 01$ & 3,72 & 26,55 \\
\hline $2010 / 85$ & $(36,65)$ & 661,30 \\
\hline
\end{tabular}

*Población 15 años y más.

\section{Elasticidad de la demanda de cigarrillos}

En el año 2010 el consumo per cápita de cigarrillos descendió $12,0 \%$ con un aumento de precio de 7,97 \%. El estudio realizado en el año 2005 mostró una fuerte relación entre las variables consumo de cigarrillos y el precio, con un coeficiente de correlación de $-0,82549$, aunque esta relación no se ha manifestado siempre debido a otras situaciones económicas y sociales que han estado asociadas con el precio relativo del cigarrillo. ${ }^{7}$

En las economías de mercado bien establecidas, un aumento en los precios del 10 $\%$ da como resultado una disminución en el consumo del 2,5 al $5 \%$. ${ }^{13}$ Aunque no es posible considerar que en el momento en que se realizó el estudio la economía de mercado en el país clasificara como tal, si se venían produciendo modificaciones que coadyuvaron a que el precio jugara el papel de mecanismo regulador del consumo (tabla 7), (figura). 


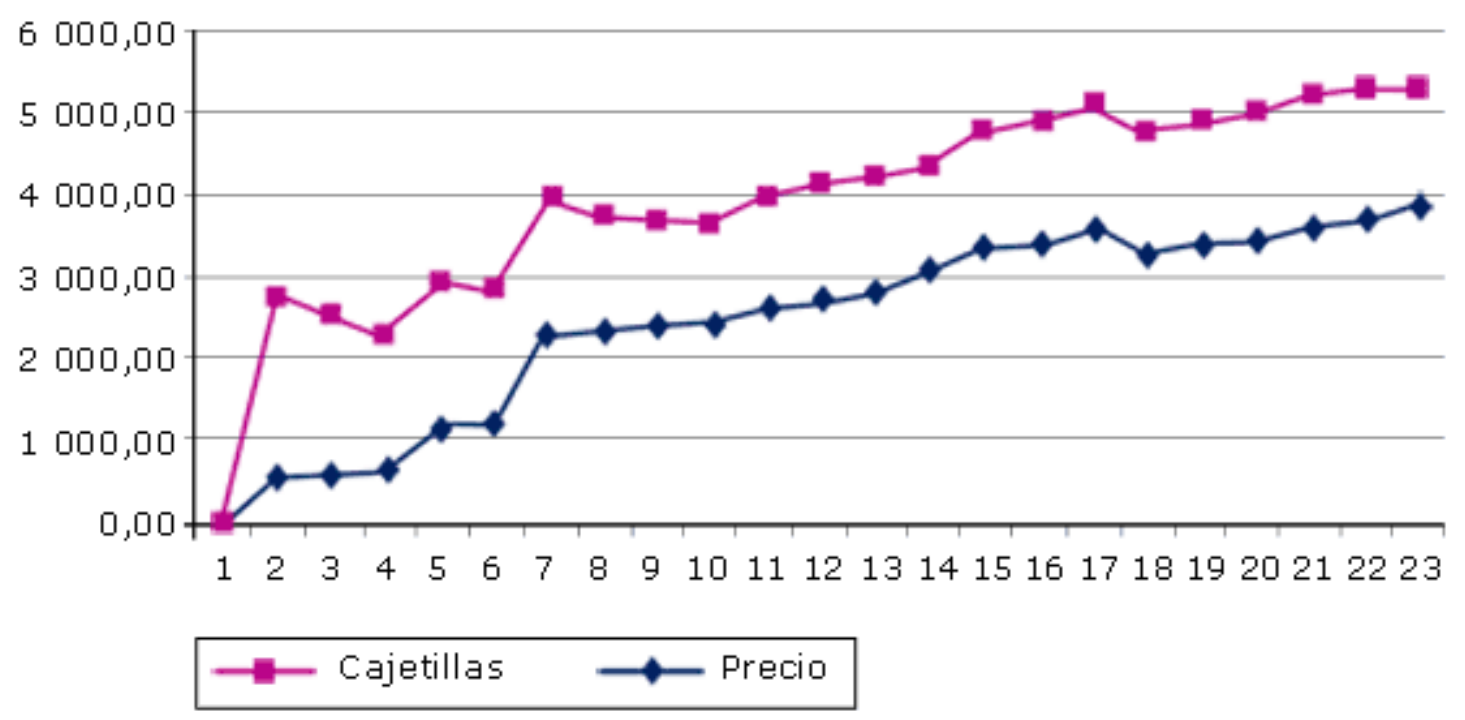

Fig. Relación cajetillas de cigarrillos per cápita y precio promedio del cigarrillo. Cuba 1985-2010.

Derivado de lo anterior, se comprobó en diferentes momentos que los cigarrillos en el mercado cubano no tenían elasticidad de la demanda, ya que no siempre se manifestó la disminución de su consumo a medida que aumentaba su precio. No ha resultado posible estudiar la elasticidad cruzada.

En el año 2010 el coeficiente encontrado fue de -1,01 que al ser ligeramente menor a - 1 mostró que la cantidad demandada de cigarrillos disminuía proporcionalmente más de lo que aumentaba el precio, esto es, se demostró elasticidad de la demanda, lo que se hace visible en la tabla 7. Un aumento del $10 \%$ en el precio del cigarrillo se estima que producirá un decrecimiento del $7 \%$ en su consumo.

En Cuba, la relación entre el incremento del precio y la reducción en la prevalencia en el período 2001 y 2010 fue de un incremento en el precio promedio del cigarrillo de $26,55 \%$ para un decrecimiento de la prevalencia de $8,2 \%(31,9 \%$ versus $23,7 \%) *$. De lo anterior se puede estimar que un incremento del $10 \%$ en el precio del cigarrillo producirá un decrecimiento de $3 \%$ en la prevalencia del tabaquismo en la población cubana de 15 años y más.

\section{DISCUSIÓN}

La disminución de las ventas de cigarrillos en el año 2010 está estrechamente relacionada con el aumento del precio promedio del cigarrillo, además del incremento de precios de otros productos, entre ellos los alimenticios. El cigarrillo compite con otras preferencias reales de consumo; la diversificación de la oferta tiende a la modificación en la elección de prioridades en el consumo.

Se une a ello los cambios en la política económica que incluyen el cese de la distribución de los productos de higiene personal y familiar que se ofertaban, aunque no de manera estable, por la tarjeta de racionamiento, el alza del precio del combustible y otros incrementos que de manera gradual y en muchas ocasiones prácticamente imperceptibles, se han ido introduciendo en el mercado. 
Otro aspecto a considerar es la situación del mercado laboral con el cese del vínculo a trabajadores, que aunque tienen la opción de derivar al sector privado, es un proceso que no siempre se puede realizar de manera simultánea.

En el 2010, los cubanos estaban preocupados por la situación económica nacional, familiar y personal y más aún con un sentimiento de incertidumbre hacia el futuro, aunque las autoridades cubanas siempre recalcan que "nadie quedará desamparado", al ser una situación diferente a la de momentos anteriores, es de suponer que no se encontraban, desde el punto de vista psicológico, con los suficientes recursos para afrontarla.

La situación social y económica descrita pudiera justificar la reducción del consumo de cigarrillos en este momento, en que por primera vez se produce la concordancia de las campañas educativas con el elemento económico para lograr el impacto que la literatura describe desde hace muchos años. ${ }^{6}$

El Convenio Marco para el Control del Tabaco en su artículo 6 hace referencia a disminuir la accesibilidad y minimizar el consumo mediante el incremento de impuestos, ${ }^{14}$ ya que el aumento de los precios es considerado el método más eficaz para reducir el consumo de tabaco en diferentes segmentos de la población, siendo especialmente sensibles los jóvenes y las personas con ingresos bajos. En Estados Unidos, el $43 \%$ de los neoyorquinos fumadores en el año 2002 que dejaron de fumar en el año 2003, manifestó que el aumento en los precios fue la razón principal. ${ }^{13}$

Elevar el precio de los cigarrillos induce a los consumidores actuales a que dejen de fumar, que los exfumadores no vuelvan a hacerlo, reduce el consumo en los que continúan fumando y evita que los posibles consumidores empiecen a fumar, lo que aumenta la presión social de otros miembros de la familia sobre el fumador para que deje de fumar.

Es interesante también considerar como la relación calidad-precio se manifiesta en el desplazamiento hacia el mercado liberado en moneda libremente convertible, pues aunque el cigarrillo que se oferta de más demanda, Popular, tiene un precio que duplica al que se oferta de forma liberada en moneda nacional, el único incremento en volumen de ventas se observó en el mercado liberado en moneda libremente convertible.

También puede explicar este desplazamiento el valor de signo ${ }^{15}$ que tienen en la población, fundamentalmente joven, aquellos productos que se ofertan en la red en divisas, pues representan un mayor estatus social y es precisamente en este grupo de edad donde se concentra la mayor fuerza laboral que recibe remuneración salarial en este tipo de moneda, unido al incremento de las posibilidades de una pequeña parte de la población al presentarse una apertura a fuentes de empleo privadas con recaudación en moneda convertible.

Al hacer un análisis de los incrementos en el mercado liberado en moneda libremente convertible, hay que tomar en cuenta que además del incremento en el período que se analiza, hay un porcentaje de incremento acumulado, por lo que en este proceso se debe producir un límite relativo al crecer anualmente el dato y complementar el volumen total de consumo actual de la población. 
Aunque no se considera en el presente análisis la elasticidad cruzada del cigarrillo como producto en el mercado, el nivel y tendencia de los ingresos monetarios y su aplicación en los diferentes conceptos de gastos de la población, dada la diversificación de la oferta, no se puede descartar la posibilidad de una reducción de la demanda global, aunque el cigarrillo a precio bajo desaparezca de la oferta.

Un estudio del Banco Mundial en el año 2009, sobre el porcentaje del monto de dinero que los grupos familiares más pobres gastan en tabaco, encuentra en Bangla Desh $1,5 \%$, en India $4 \%$, Sudáfrica $4,7 \%$, Bulgaria $10,4 \%$ y en Indonesia 15 $\% .{ }^{16}$ Aunque no se dispone de un estudio similar en Cuba, en una investigación realizada en Ciudad de La Habana en el año 2009, se halla que los gastos medios de bolsillo de los fumadores fueron más altos en comparación con los de los no fumadores en todos los rubros analizados, a lo que se le adiciona que la relación de gastos por pérdidas laborales resulta casi el doble en fumadores que en no fumadores, lo que repercute en una disminución mayor de los ingresos. (Gálvez González AM. Costos de atención a la salud asociados al tabaquismo en municipios de Ciudad de La Habana, 2009. Escuela Nacional de Salud Pública. La Habana; 2010).

En este contexto, en la medida en que la balanza de ingresos y gastos se incline hacia los gastos, se unirá a los efectos de la labor educativa sobre la nocividad del tabaquismo, del tabaquismo pasivo y al control de las prohibiciones de fumar y favorecerá una mayor asociación de estas acciones, que con anterioridad no tenían la coherencia y complementariedad que se requiere en un programa de prevención y control.

En el 2011, el decrecimiento del consumo de cigarrillos debe ser superior debido al incremento del precio promedio del cigarrillo y porque a largo plazo el impacto es mayor debido a que los consumidores adictos reaccionan con el tiempo a los aumentos permanentes de precios. Lo anterior permite alertar sobre la posible pérdida progresiva de la capacidad recaudadora del cigarrillo en la economía interna.

También se debe tomar en consideración por las autoridades competentes, que de continuar la distribución gratuita, o a precios bajos a los organismos, existe la posibilidad de un intento de comercio ilegal de cigarrillos ofertando el producto a un precio menor que el de la red oficial.

La literatura refiere que aproximadamente la mitad del impacto que tiene el precio en el consumo de tabaco surge de los cambios en la prevalencia debidos en gran parte al abandono del hábito de fumar de consumidores actuales. Un aumento de $10 \%$ en el precio reduce la prevalencia entre 1 y $2,5 \%$ en países de altos ingresos y entre 2,5 y $5 \%$ en países de bajos y medianos ingresos. Los cálculos realizados en Estados Unidos sugieren que al aumentar el precio el $10 \%$, la cantidad de fumadores que intentan dejar de fumar aumenta en más del $10 \%$, de este porcentaje, el $2 \%$ lo logrará a largo plazo. ${ }^{13}$

En Cuba se pudo estimar que un incremento de $10 \%$ en el precio del cigarrillo producirá un decrecimiento de $3 \%$ en la prevalencia del tabaquismo en la población cubana de 15 años y más, similar al estimado para los países de bajos y medianos ingresos. La literatura refiere también que la elasticidad de la prevalencia representa aproximadamente la mitad de la elasticidad del consumo, ${ }^{13}$ datos que concuerdan con el estimado obtenido de un decrecimiento de $7 \%$ en el consumo. 
El Programa para la Prevención y Control del Tabaquismo debe reforzar su componente educativo con información sobre técnicas de cesación y a la vez aumentar las ofertas de tratamiento y rehabilitación en las instituciones sanitarias con el propósito de facilitarles esa opción a los fumadores interesados en dejar de fumar.

Sin embargo, no se puede olvidar, que ya en el 2012 el precio promedio del cigarrillo no tendrá modificaciones, ya que los aumentos se producían por la venta a precios bajos en el mercado normado, por lo que para lograr cambios en el consumo y por consiguiente en la prevalencia, será necesario prever el incremento progresivo del precio en los dos mercados si se quiere reducir el tabaquismo como factor de riesgo.

Volverá de nuevo a mostrarse la ambivalencia en intereses en la definición de las políticas públicas en lo que respecta al tabaquismo, si la economía o la salud pública, porque la venta de cigarrillos contribuye en gran medida al equilibrio de las finanzas internas del país pero contribuye también a incrementar la morbilidad y mortalidad de la población.

La salud pública tiene una oportunidad para reforzar sus acciones en función de mejorar el bienestar de la población mediante la reducción del tabaquismo, importante factor de riesgo en la actual situación de salud y de esta forma cumplir las proyecciones de la salud pública para el año 2015 . $^{17}$

El estudio realizado permite concluir que la reducción del consumo de cigarrillos ratifica la importancia del precio en los programas de prevención y control del tabaquismo; que el incremento del precio promedio del cigarrillo inicia una tendencia creciente con un importante aumento para el año 2011, cuando ya no se ofertarán cigarrillos en el mercado racionado a precios bajos, pero la presencia de fuentes de empleo privadas, van a generar ingresos en moneda libremente convertible en la población que pueden atenuar los actuales efectos de la elevación en el precio de los cigarrillos; la elasticidad de la demanda de cigarrillos se verá beneficiada con el alza de precios de los productos alimenticios en el mercado mundial y por tanto en el nacional, lo que produce una mayor elasticidad cruzada hasta ahora presente, pero no estudiada.

Finalmente se considera importante que el Programa de Prevención y Control del Tabaquismo centre su estrategia en reforzar las acciones que aumenten la asociación de las medidas económicas favorecedoras a la reducción del consumo de cigarrillos en la población y en particular en los jóvenes, con las medidas educativas.

Queda la interrogante de hacia dónde se inclinará de nuevo la balanza: ¿tabaco o salud?

\section{REFERENCI AS BI BLI OGRÁFICAS}

1. López Casasnovas G, Marc Sáez M. A vueltas con el aseguramiento sanitario privado. Barcelona. Gaceta Sanitaria. 2005; 19(1).

2. Suárez Lugo N. Paradojas, controversias, discurso y realidad del tabaquismo en Cuba. Revista Cubana Salud Pública [Internet]. 2011 [citado 24 Feb 2010];37(1). 
Disponible en: $\quad$ http://scielo.sld.cu/scielo.php?script=sci_arttext\&pid=S086434662011000100010\&lng=es\&nrm=iso\&tIng=es

3. Oficina Nacional de Estadísticas. Salario medio en cifras. Cuba 2010. La Habana: ONE; 2011.

4. Ravsberg F. Cuba, paraíso de los fumadores. BBC Mundo [Internet]. 2011 [citado 24 Feb de 2011]. Disponible en: http://www.bbc.co.uk/mundo/noticias/2011/02/110223_cuba_tabaco_sao.shtml

5. Gaceta Oficial República de Cuba. No. 022 Extraordinaria. 27 de agosto de 2010. R. No. 244/10. La Habana: Ministerio de Finanzas y Precios; 2010.

6. Suárez Lugo N. Tabaco o Salud: una decisión social. Revista Horizontes Sanitarios. UJAT México. 2004; 3(2).

7. Suárez Lugo N. El consumo de productos manufacturados del tabaco en Cuba. Rev Cubana Salud Pública [Internet]. 2006 [citado 24 Feb 2010];32(2). Disponible en: $\quad$ http://scielo.sld.cu/scielo.php?script=sci_arttext\&pid=S0864$\underline{34662006000200002 \& \operatorname{lng}=e s \& n r m=i s o \& t \operatorname{lng}=e s}$

8. Ministerio de la Agricultura. TABACUBA. Empresa ATM. Consumo Nacional de Cigarrillos y Tabacos. 2010. La Habana: MINAGRI; 2011.

9. Ministerio de la Agricultura. Grupo TABACUBA. BRASCUBA. Ventas internas de cigarrillos. 2010. La Habana: MINAGRI; 2011.

10. Oficina Nacional de Estadísticas. Anuario Estadístico 2010. Edición 2010 [Internet]. La Habana: ONE; 2011 [citado 2 Mar 2011]. Disponible en: http://www.one.cu/sien2010.htm

11. Chaloupka FJ. Una Mirada hacia el Futuro. Resumen de las Conclusiones más Importantes. Global Tobacco Control-Learning from the Experts [Internet]. Baltimore; School of Public Health Johns Hopkins Bloomberg [citado 15 Feb 2010]. Available from: http://www.GlobalTobaccoControl.org/es

12. Suárez Lugo N. Enfoque social de la mercadotecnia sanitaria. La Habana: Editorial Ciencias Médicas; 2007.

13. Chaloupka FJ. Aplicaciones de impuestos al tabaco. Global Tobacco ControlLearning from the Experts [Internet]. Baltimore; School of Public Health Johns Hopkins Bloomberg [citado 15 Feb 2010]. Available from: http://www. GlobalTobaccoControl.org/es

14. Organización Mundial de la Salud. Convenio Marco para el Control del Tabaco. Ginebra: Organización Mundial de la Salud; 2003.

15. Suárez Lugo N. El consumo y la salud. Cali, Colombia: Editorial CATORSE; 2010. 
16. Waters H. Introducción a la economía del control del tabaco. Global Tobacco Control-Learning from the Experts [Internet]. Baltimore; School of Public Health Johns Hopkins Bloomberg [citado 15 Feb 2010]. Available from: http://www. GlobalTobaccoControl.org/es

17. Ministerio de Salud Pública. Proyecciones de la salud pública en Cuba para el año 2015. La Habana: Editorial Ciencias Médicas; 2006.

Recibido: 21 de julio de 2011.

Aprobado: 16 de septiembre de 2011.

Nery Suárez Lugo. Escuela Nacional de Salud Pública. Calle 100 No. 1132 e/ E y Perla. Altahabana, Municipio Boyeros. La Habana, Cuba.

Correo electrónico: nerysl@infomed.sld.cu

* Instituto Nacional de Higiene y Epidemiología. Encuesta Nacional de Factores de Riesgo 2010. Informe Preliminar. INHEM. Ministerio de Salud Pública. La Habana, mayo 2011. 